\title{
Haploinsufficiency of MBD5 associated with a syndrome involving microcephaly, intellectual disabilities, severe speech impairment, and seizures
}

\author{
Stephen R Williams ${ }^{1}$, Sureni V Mullegama ${ }^{1}$, Jill A Rosenfeld ${ }^{2}$, Aditi I Dagli ${ }^{3}$, Eli Hatchwell ${ }^{4}$, William P Allen ${ }^{5}$, \\ Charles A Williams ${ }^{3}$ and Sarah H Elsea ${ }^{\star, 1,6}$
}

\begin{abstract}
Microdeletion of chromosome 2q23.1 results in a novel syndrome previously reported in five individuals. Many of the del(2)(q23.1) cases were thought to have other syndromes such as Angelman, Prader-Willi, or Smith-Magenis because of certain overlapping clinical features. We report two new cases of the 2q23.1 microdeletion syndrome, describe the syndrome phenotype, define the minimal critical region, and analyze the expression of critical region genes toward identification of the causative gene(s) for the disorder. Individuals with del(2)(q23.1) have severe developmental and cognitive delays, minimal speech, seizures, microcephaly, mild craniofacial dysmorphism, behavioral disorders, and short stature. The deletions encompassing 2q23.1 range from $>4 \mathrm{Mb}$ to $<200 \mathrm{~kb}$, as identified by oligonucleotide and BAC whole-genome array comparative hybridization. The minimal critical region includes a single gene, MBD5, deleted in all cases, whereas all but one case also include deletion of EPC2. Quantitative real-time PCR of patient lymphoblasts/lymphocytes showed an $\sim 50 \%$ reduced expression of MBD5 and EPC2 compared with controls. With similar phenotypes among the 2q23.1 deletion patients, the idea of one or more common genes causing the pathological defect seen in these patients becomes evident. As all five previous cases and the two cases in this report share one common gene, MBD5, we strongly suspect that haploinsufficiency of MBD5 causes most of the features observed in this syndrome.
\end{abstract}

European Journal of Human Genetics (2010) 18, 436-441; doi:10.1038/ejhg.2009.199; published online 11 November 2009

Keywords: developmental delay; microcephaly; seizures; 2q23.1; microdeletion; MBD5

\section{INTRODUCTION}

Owing to the phenotypic complexity of many genomic disorders and the fact that most of these disorders result in developmental delays and behavioral problems, overlapping physical and behavioral features between syndromes are commonly observed. ${ }^{1}$ Although there are clear phenotypic differences between most syndromes, Smith-Magenis, Down, and Prader-Willi syndromes have many major features in common, including craniofacial features, obesity, and hypotonia, whereas Angelman and Rett syndromes feature seizures and severe developmental delays. Correct identification and diagnosis of a specific syndrome are more difficult because of these phenotypic similarities. Ultimately, when comparative genomic hybridization (CGH) data are considered and new syndromic regions of the genome are identified, sorting these cases by phenotype alone may be even more difficult.

Microdeletion of chromosome 2 q23.1 results in a novel syndrome previously reported in five individuals. ${ }^{2-6}$ In this study, we report two new cases with overlapping 2q23.1 deletions and draw attention to possible candidate genes that could be causative for the characteristic phenotype observed in our cases and in the other five patients reported in literature. A comparison of phenotypes to the other five known cases revealed similar features and confirmed a consistent phenotypic pattern associated with 2 q23.1 microdeletion. Although this new microdeletion syndrome has certain phenotypic similarities to various known disorders such as Angelman, Smith-Magenis, and Rett syndromes, it is not a classic phenocopy for any of these conditions. On the basis of our evaluation of the spectrum of clinical features in our cases and in previously reported ones, we have further delineated the clinical characterization of this new microdeletion syndrome. On the basis of the similar features observed, we established a clinical characterization of the novel microdeletion syndrome. Furthermore, an analysis of all reported cases involving a deletion of 2q23.1 revealed a minimal critical region consisting of a single gene, $M B D 5$, as the only deleted gene in one case, and MBD5 and EPC2 deleted in all other cases. In this study, we report two new cases of the 2 q23.1 deletion syndrome, describe the syndrome phenotype, define the minimal critical region, and analyze the expression of critical region genes toward identification of the causative gene(s) for the disorder.

\section{METHODS}

Patient ascertainment

Subjects were referred for molecular evaluation of the Smith-Magenis syndrome (SMS185) and the Prader-Willi syndrome (SMS361). The Institutional Review Boards at Virginia Commonwealth University and Michigan State University approved this study.

${ }^{1}$ Department of Human and Molecular Genetics, Virginia Commonwealth University School of Medicine, Richmond, VA, USA; 2 Signature Genomic Laboratories, Spokane, WA, USA; ${ }^{3}$ RC Philips Unit, Division of Genetics and Metabolism, Department of Pediatrics, University of Florida College of Medicine, Gainesville, FL, USA; ${ }^{4}$ Department of Pathology, State University of New York, Stony Brook, NY, USA; ${ }^{5}$ Fullerton Genetics Center, Mission Hospital, Asheville, NC, USA; ${ }^{6}$ Department of Pediatrics, Virginia Commonwealth University School of Medicine, Richmond, VA, USA

*Correspondence: Dr SH Elsea, Department of Pediatrics, Virginia Commonwealth University School of Medicine, Richmond, VA 23298, USA.

Tel: +1 804-628-0987; Fax: +1 804-628-1609; E-mail: selsea@vcu.edu

Received 18 June 2009; revised 6 October 2009; accepted 7 October 2009; published online 11 November 2009 


\section{Cell lines}

Lymphoblastoid cell lines (Epstein-Barr virus-transformed human lymphocytes) from SMS185, her mother (SMS184), her father (SMS183), and from an unaffected control were cultured in RPMI-1640 with $2 \mathrm{~mm}$ L-glutamine and $25 \mathrm{~mm}$ HEPES (4-(2-hydroxyethyl)-1-piperazineethane-sulfonic acid) (Invitrogen, Carlsbad, CA USA), 10\% fetal bovine serum, and 1\% penicillin/ streptomycin antibiotic-antimycotic solution (Invitrogen) at $37^{\circ} \mathrm{C}$ in a humidified $5 \% \mathrm{CO}_{2}$ chamber. Cells were grown at the same time period for a span of 3 weeks and supplemented with fresh media every 2-3 days as needed. Cells were monitored for growth using bright field microscopy and were counted using Trypan blue exclusion with a hemocytometer. After a 3-week growth period, the cells were counted, pelleted, and used immediately for RNA extraction or stored at $-80^{\circ} \mathrm{C}$ for RNA extraction at a later date.

\section{RNA extraction}

RNA was extracted from either $5-10 \times 10^{6}$ lymphoblast cells using the standard TRIzol protocol (Invitrogen) or from $4 \mathrm{ml}$ whole blood, as described here. The whole blood was mixed with $46 \mathrm{ml}$ Puregene RBC lysis solution, mixed, and let to stand at room temperature for $15 \mathrm{~min}$. White blood cells were pelleted at $600 \mathrm{~g}$ for $10 \mathrm{~min}$ at $4{ }^{\circ} \mathrm{C}$. The pellet was resuspended in $1 \mathrm{ml} \mathrm{RBC}$ lysis solution and incubated at room temperature for $5 \mathrm{~min}$. Cells were pelleted at 3000 r.p.m. at $4{ }^{\circ} \mathrm{C}$ for $2 \mathrm{~min}$ resuspended in $1 \mathrm{ml}$ cold PBS, and further pelleted at 3000 r.p.m. at $4{ }^{\circ} \mathrm{C}$ for $2 \mathrm{~min}$. RNA was then isolated using the standard TRIzol protocol (Invitrogen). The concentration and purity of RNA were measured at an absorbance of 260 and $280 \mathrm{~nm}$. RNA was stored at $-80^{\circ} \mathrm{C}$ until ready for use.

\section{Real-time qRT-PCR}

First-strand cDNA synthesis using SuperScript II RT (Invitrogen) was prepared with $3 \mu \mathrm{g}$ of total RNA using Olgio(dT) $)_{12-18}$, and optional RNase OUT treatment, followed by RNA degradation with 2.5 units RNase H (Applied Biosystems Inc., Foster City, CA, USA). For quantitative real-time PCR, predesigned assays on Demand Gene Expression Products (Fermentus, Glen Burnie, MD USA) Taqman MGB probes for MBD5, EPC2, KIF5C, and GAPDH were used (Applied Biosystems Inc.). GAPDH was used as the endogenous control. All samples of cDNA were run in triplicate in $10 \mu \mathrm{l}$ reaction volumes. Taqman Universal PCR Master Mix (Applied Biosystems Inc.), the probe, and deionized water were mixed together in a fixed ratio, and $8 \mu \mathrm{l}$ was added to each well. Diluted cDNA (1:5) was then added to each well. PCR conditions were the Standard 7500 Run mode of the ABI Prism 7900 HT Sequence Detection System (Applied Biosystems Inc.). The cycle threshold (CT) was determined during the geometric phase of the PCR amplification plots, as recommended by the manufacturer. Relative differences in transcript levels were quantified using the $\Delta \Delta \mathrm{Ct}$ method. Acquired data were analyzed using 7500 Fast System SDS Software (Applied Biosystems Inc.).

\section{RAI1 sequencing}

RAI1 sequencing was performed as previously described ${ }^{7}$ for SMS185. A novel familial polymorphism was found, but no pathogenic mutations were identified in this case.

\section{DNA extraction}

Peripheral blood was collected, from which DNA was prepared immediately or from cell lines established previously. DNA was extracted following standard phenol chloroform methods.

\section{Whole-genome array comparative genomic hybridization}

BAC aCGH was performed as previously described. ${ }^{8,9}$

\section{Oligonucleotide array CGH}

Oligonucleotide-based microarray analysis was performed for SMS361 using a 105K-feature whole-genome microarray (SignatureChip Oligo Solution made for Signature Genomic Laboratories by Agilent Technologies, Santa Clara, CA, USA) as previously described. ${ }^{10}$

Oligonucleotide-based microarray analyses were performed using a $244 \mathrm{~K}$ feature whole-genome microarray made for Signature Genomic Laboratories by
Agilent Technologies to characterize the extent of the abnormalities in SMS185 and SMS361. Genomic DNA labeling was performed as described for BAC arrays, whereas array hybridization and washing were performed as specified by the manufacturer (Agilent Technologies). Arrays were scanned using an Axon 4000B scanner (Molecular Devices, Sunnyvale, CA, USA) and analyzed using Agilent Feature Extraction software v9.5.1 and Agilent CGH Analytics software v3.5.14. Results were then displayed using custom oligonucleotide array CGH analysis software (Oligoglyphix, Signature Genomic Laboratories, Spokane, WA, USA).

\section{Fluorescence in situ hybridization (FISH) analysis}

Metaphase FISH was performed using bacterial artificial chromosome (BAC) clone RP11-951G8 from the 2q deleted region as previously described. ${ }^{11}$ FISH data are shown in Supplementary Figure 1.

\section{RESULTS}

Case reports

Case 1 (SMS185). SMS185 is a 13-year-old Caucasian girl first seen for a clinical genetic evaluation when she was 4 years old because of concerns regarding developmental delays, short stature, and microcephaly (Table 1, Figure 1, Supplementary Figure 2). She was the first child of phenotypically normal and nonconsanguineous parents (mother 25 years and father 19 years). No remarkable detail of pregnancy was mentioned. Birth weight was $3.0 \mathrm{~kg}$ and length was $48.26 \mathrm{~cm}$ (both between the 25th and 50th percentile) with no perinatal problems. As an infant, she had significant feeding difficulties due to hypotonia. An atrial septal defect was identified at 1 year of age, with spontaneous closure by 3 years of age. By 16 months of age, microcephaly, global developmental delays, and short stature were evident. A pediatric developmental evaluation was pursued by 3 years for sleep difficulties and hyperactivity.

The initial physical examination at age $4 \frac{5}{12}$ years revealed a weight of $16.1 \mathrm{~kg}$ (50th percentile), a height of $92.25 \mathrm{~cm}$ (just below the third percentile), and a head circumference of $46 \mathrm{~cm}$ ( three SD below the mean). She was quite active, with a hoarse voice and no understandable words. Craniofacial examination revealed midface hypoplasia, an upturned nose, apparently widely spaced eyes, and a tented upper lip (Figure 1, Table 1, Supplementary Figure 2). Skeletal examination revealed short, thick, and tapered fingers, which measured 9.5, 5.5, and $4 \mathrm{~cm}$ for total hand, palm, and middle finger lengths, respectively (all below the third percentiles). $\mathrm{X}$-rays of the hands revealed shortened and widened metacarpals and phalanges in a symmetric manner, with no evidence of focally shortened bones.

Follow-up exam at $9 \frac{3}{12}$ years revealed a history of the onset of complex-partial seizures at 8 years associated with an abnormal EEG with 'mild, diffuse encephalopathy' changes noted, but no areas of focal changes or seizure activity. Her brain MRI scan was normal, and after beginning Trileptal, there was no recurrence of seizure activity. A further evaluation at $10 \frac{9}{12}$ years noted behavioral difficulties related to significant hyperactivity, minimal expressive speech, a weight of $33.1 \mathrm{~kg}$ (30th percentile), a height of $123.2 \mathrm{~cm}$ (approximately three SD below the mean), and a head circumference of $50 \mathrm{~cm}$ (just below the third percentile). Her physical features were unchanged from the initial examination.

Results of karyotype and FISH analyses to detect a Smith-Magenis syndrome deletion were normal at 4 years of age. Sequencing of the RAI1 gene did not reveal any pathogenic mutations (data not shown). DNA methylation for the Angelman syndrome was normal at 9 years.

Case 2 (SMS361). SMS361 is a 10-year-old Hispanic girl who was first seen at 26 months of age for developmental delay (Figure 1). She 
Table 1 Clinical features of cases with 2q23.1 microdeletion syndrome

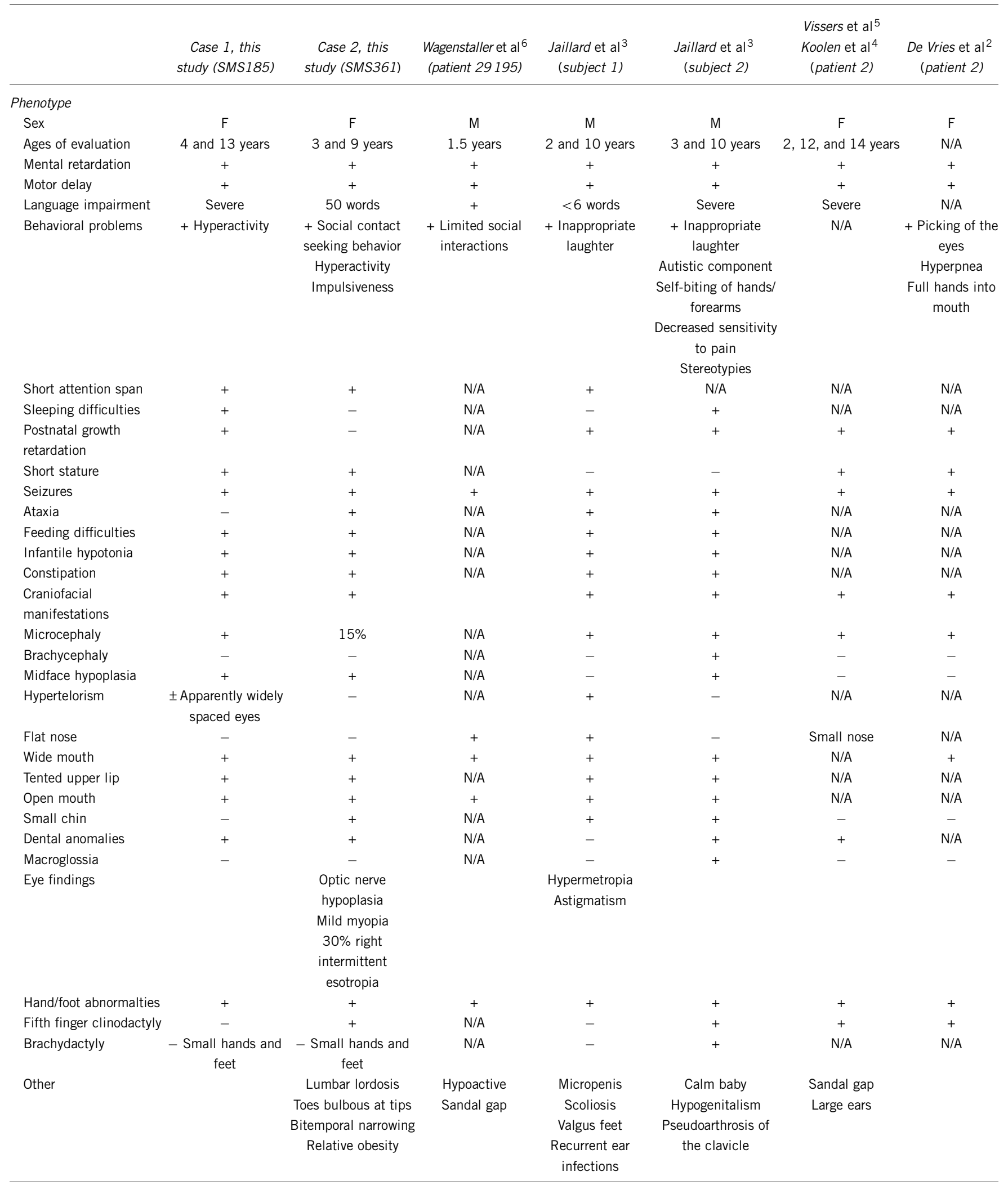

\footnotetext{
+ , Feature present; -, feature absent; N/A, unknown.
} 


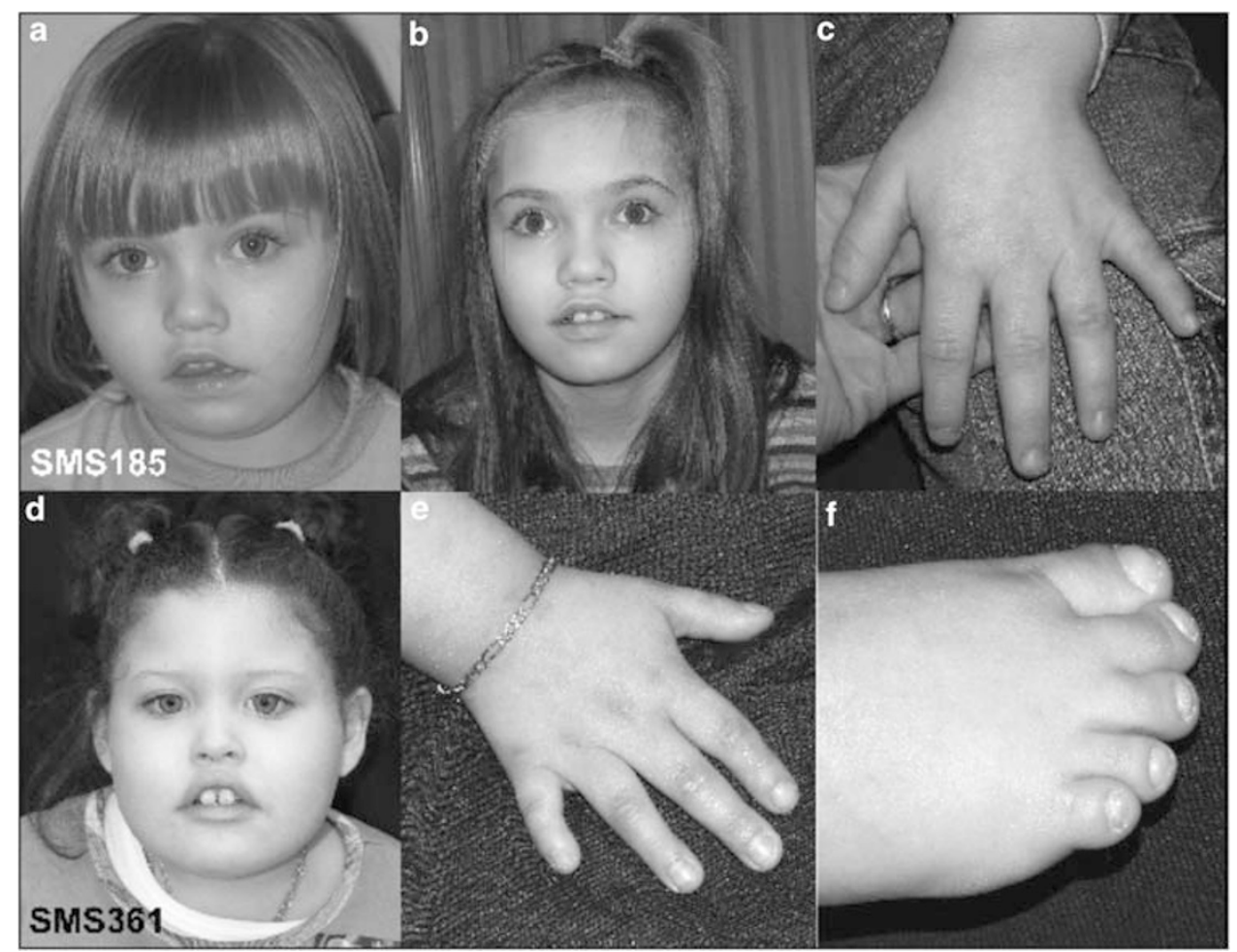

Figure 1 Clinical features of the 2q23.1 deletion syndrome. (a) Case 1, SMS185 aged 4 years. Note tented upper lip, open mouth, and microcephaly. (b) SMS185, 13 years of age, note tented upper lip and prominent incisors. (c) Hands of SMS185, 13 years of age; note brachydactyly. (d) Case 2, SMS361 aged 8 years. Note tented upper lip with prominent upper incisors and open mouth. (e) Hand of SMS361. Note small hand. (f) Foot of SMS361, note small foot and short toes with bulbous tips.

was born to an 18-year-old gravida 1 mother. She was thought to have Down syndrome at birth, but chromosome testing was negative by report. Her family history was unremarkable.

She had a generalized tonic-clonic seizure at 8 months of age, but her EEG and CT scans were normal. She was treated with valproic acid and has had only 1-2 seizures/year. At 4 years of age, a repeat EEG showed spike and wave epileptiform discharges from the frontal lobes. This pattern has been observed on and off during successive EEGs, with the latest EEG at 7 years of age demonstrating a left-sided spike and wave pattern from the left frontal lobe. Several brain MRIs were normal.

She walked after 2 years of age. Her speech was delayed, but she did learn new words. At the age of 2 years and 11 months, she knew about a total of 50 words in English and Spanish. She did not start putting two words together until after 9 years of age. Her speech was unclear, although she continues to add words to her vocabulary. She can follow simple commands, but her attention span is limited.

A physical examination at 9 years of age showed truncal obesity, with a height of $122 \mathrm{~cm}$ ( fifth percentile), weight of $41.0 \mathrm{~kg}$ (90th percentile), and an occipitofrontal circumference of $50.5 \mathrm{~cm}$ (15th percentile). She was hyperactive, impulsive, and had a short attention span, moving from object to object in the examination room (Table 1). She had social contact-seeking behavior, maintained good eye contact, and had a happy demeanor. She had an unusual facial appearance with a tented upper lip and bitemporal narrowing. She had light-colored irides. Her hands were small (total hand length of $13 \mathrm{~cm}$, less than third percentile) and showed clinodactyly of the fifth digits (although her hand X-ray showed no shortened fourth metacarpal). Her total foot length was $17.5 \mathrm{~cm}$ (less than third percentile) and the toes were bulbous at the tips (Figure 1). Ophthalmologic examination revealed mild optic nerve hypoplasia, mild myopia, and a $30 \%$ right intermittent esotropia. Her caretaker reports a healthy appetite but no food seeking was described.

\section{Molecular analyses}

Array comparative genomic hybridization. SMS185 was evaluated by whole-genome array comparative hybridization (aCGH BAC array), ${ }^{12}$ revealing a $\sim 700-\mathrm{kb}$ deletion of 2 q23.1 and confirmed by a clinical BAC array (data not shown). To further refine the break points of this deletion, a high-resolution oligo array (Agilent 244K) was performed, defining the deletion region to be $\sim 930 \mathrm{~kb}$ at chr2:148 $447496-$ 149377297 (hg18 coordinates, Figure 2), encompassing four known genes, ORC4 L, MBD5, EPC2, and KIF5C.

SMS361 was evaluated by both $105 \mathrm{~K}$ and $244 \mathrm{~K}$ oligo arrays (Figure 2), as described in the Methods section. Array data revealed a 3.51-Mb deletion of chromosome 2q22.3-q23.3 (chr2:146 798229 150310317 , hg18 coordinates) encompassing nine known or predicted genes, PABPCP2, ACVR2A, ORC4L, MBD5, EPC2, KIF5C, LYPD6B, LYPD6, and MMADHC.

Metaphase FISH with RP11-951G8 confirmed a deletion in five out of five cells examined for both SMS185 and SMS361 (Supplementary Figure 1). Array data indicate a common, overlapping region between these two cases that includes ORC4L, MBD5, EPC2, and KIF5C (Figure 2). 


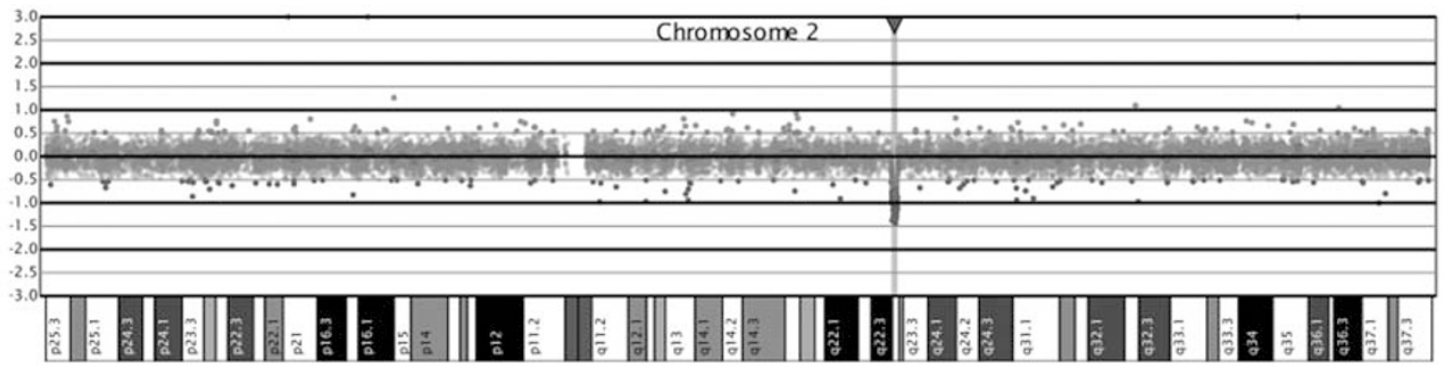

b

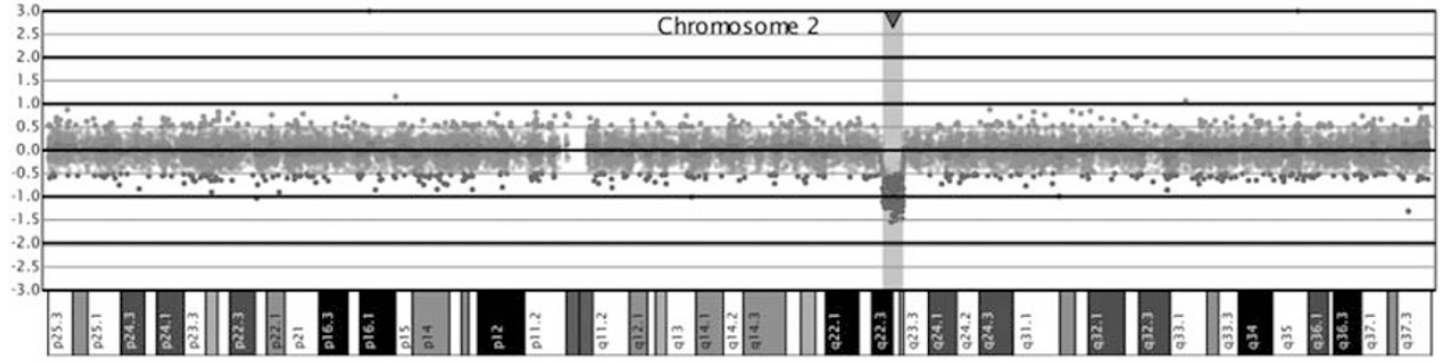

C

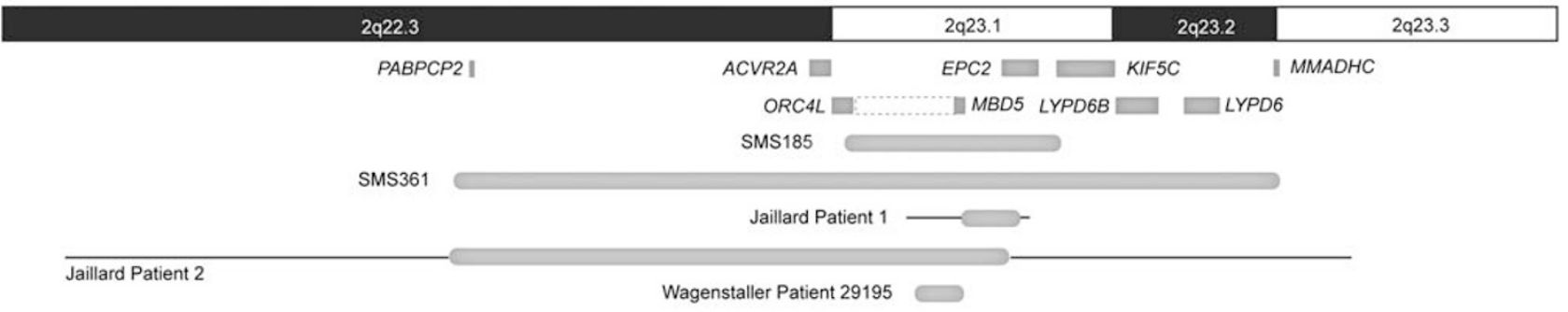

De Vries Patient 2

Koolen Patient 1

Figure 2 Array analysis of individuals with microdeletions of 2q23.1. (a, b) Oligonucleotide microarray profiles for (a) a single-copy loss of $930 \mathrm{~kb}$ at chr(2)(q23.1); (chr2: 148447496-149377297) in SMS185 and (b) a single-copy loss of $3.51 \mathrm{Mb}$ at chr(2)(q22.3-q23.3); (chr2: 146798229$150310317)$ in SMS361. For microarray plots, probes are ordered on the $x$ axis according to physical mapping positions, with distal $2 p$ to the left and distal $2 \mathrm{q}$ to the right. (c) Summary of the deletion sizes in individuals with microdeletions encompassing 2q23.1. Green bars indicate the minimum deletion sizes in individuals in this study and those in the literature, and black lines extend to show maximum deletion sizes. Genes in the region are indicated by blue boxes. Coding exons for MBD 5 are included in the solid blue box, whereas $5^{\prime}$ noncoding exons described by Wagensteller et al are within the dashed blue box. The color reproduction of this figure is available on the html full text version of the manuscript.

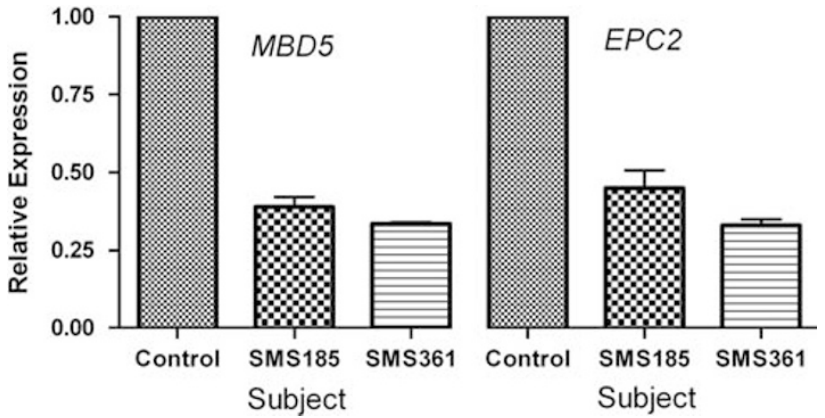

Figure 3 Quantitative real-time PCR expression analysis of MBD5 and EPC2 in patient cells. MBD5 and EPC2 relative gene expressions in SMS185 (EBV-derived lymphoblastoid cell line) and SMS361 (fresh white blood cells) are shown. Gene expression is shown relative to GAPDH expression and control cells, with relative expression values based on the $\Delta \Delta \mathrm{Ct}$ value with normal control set to 1 . Control cells were cultured or isolated at the same time and with the same methods as test cells. All experiments were performed in triplicate and average values for all combined experiments are shown $(n=2-5)$.
Quantitative real-time PCR. Loss of genes or gene function due to chromosomal deletion does not necessarily correlate to a reduction in gene expression; however, haploinsufficiency of one or more genes is typically the pathological defect in microdeletion syndromes. To determine the genes that have a reduced expression in the overlapping 2q23.1 region, both subjects were assessed for MBD5, EPC2, and KIF5C mRNA levels in white blood cells. Correlating directly to the confirmed deletions, both SMS185 and SMS361 have an $\sim 50 \%$ reduced expression of MBD5 and EPC2 mRNA compared with normal controls (Figure 3). Apparently normal levels of expression were observed for KIF5C; however, KIF5C has a very low expression in peripheral blood, and thus, levels may not be reflective of the expression in the brain (data not shown). KIF5C expression may be affected in both the larger and smaller deletion cases, as the smaller deletion includes the $5^{\prime}$-region of the KIF5C gene, which may alter gene expression. The reduction in expression of MBD5 and EPC2 supports the potential role of each of these genes in the pathophysiology of this disorder. 


\section{DISCUSSION}

In this study, we present two subjects, SMS185 and SMS361, with a $\operatorname{del}(2)(\mathrm{q} 23.1)$. These individuals have a distinct clinical phenotype that includes severe cognitive impairment, seizures, relative obesity, small hands and feet, and mild craniofacial dysmorphism, including one child with microcephaly.

A recent report described two patients with overlapping deletions of this region on chromosome 2 exhibiting a 'pseudo-Angelman' phenotype. ${ }^{3}$ Both the subjects in this study tested negative for an abnormal SNRPN DNA methylation patterning of the $15 \mathrm{q} 11.2$ region, which rules out a diagnosis of Angelman syndrome. However, they do have features consistent with the 'pseudo-Angelman' phenotype described by Jaillard et al (2008). ${ }^{3}$

In addition to the report by Jaillard et al $(2008)^{3}$, three other cases of 2 q23.1 deletion have been described ${ }^{2,4,5}$ (Figure 2). The common phenotypes observed in these subjects are severe mental retardation, motor delay, severe language impairment (sometimes accompanied by hoarse voice), behavioral abnormalities (including hyperactivity and inappropriate laughter), postnatal growth retardation, relative obesity, and seizures (Table 1). In addition, a distinctive craniofacial phenotype is also apparent that includes small head size, wide and open mouth, a tented upper lip, and prominent incisors (Figure 1). Skeletal abnormalities include generalized brachydactyly (Figure 1, Table 1).

The more variable phenotypes between the cases reported here and those in previous studies include craniofacial abnormalities such as brachycephaly and relative hypertelorism (likely associated with microcephaly), as well as eye findings and genital abnormalities. Overall, these individuals with varying overlapping deletions of 2q23.1 present with a very similar global phenotype, which leads us to believe that a common gene(s) might be involved.

It is worthwhile to mention that reported duplications of the $2 \mathrm{q} 23$ region are rare and tend to be much larger in size than the deletions described in this and previous reports. ${ }^{13-15}$ The reported duplication 2q23 cases presented with craniofacial abnormalities, low set ears, and heart, kidney, genital, skeletal, and neurological abnormalities, resulting in death in early infancy. ${ }^{16}$ The phenotype observed in $\operatorname{dup}(2)(\mathrm{q} 23)$ further supports a gene dosage as a mechanism and the importance of the genes in this region.

When considering all of the reported deletions involving 2q23.1 (Figure 2), a critical region emerges that includes a single gene, MBD5. MBD5 (methyl-CpG binding domain protein 5) is expressed neuronally and likely functions in the regulation of gene expression. ${ }^{17,18}$ MBD5 contains a methyl-binding domain, sharing sequence homology to MECP2, which when mutated or deleted, results in Rett syndrome. We have shown in this study that MBD5, in addition to $E P C 2$, is haploinsufficient in patient white blood cells and lymphoblastoid cells (Figure 3), providing further evidence of its role in this syndrome and the need for proper gene dosage for normal development and behavior. We do not, however, rule out the possibility that KIF5C and/or EPC2 may contribute to variability or severity of the syndrome. These data, taken together with the previous report of a small deletion within $M B D 5,3,6$ indicate that haploinsufficiency of MBD5 is the likely common pathological defect for most features of this syndrome.

\section{CONFLICT OF INTEREST}

The authors declare no conflict of interest.

\section{ACKNOWLEDGEMENTS}

We thank Aaron Theisen and Sara Minier (Signature Genomic Laboratories) for their help with Figure 2, Ryan Traylor (Signature Genomic Laboratories) for running the $244 \mathrm{~K}$ arrays, and Dr Santhosh Girirajan for performing RAII sequencing (VCU). This study was supported, in part, by the Fondation Jerome Lejeune (to SHE).

1 Jedele KB: The overlapping spectrum of Rett and Angelman syndromes: a clinical review. Semin Pediatr Neurol 2007; 14: 108-117.

2 de Vries BB, Pfundt R, Leisink $M$ et al: Diagnostic genome profiling in mental retardation. Am J Hum Genet 2005; 77: 606-616.

3 Jaillard S, Dubourg C, Gerard-Blanluet M et al: 2q23.1 microdeletion identified by array-CGH: an emerging phenotype with Angelman-like features? J Med Genet 2008, PMID: 18812405

4 Koolen DA, Vissers LE, Nillesen W et al: A novel microdeletion, del(2)(q22.3q23.3) in a mentally retarded patient, detected by array-based comparative genomic hybridization. Clin Genet 2004; 65: 429-432.

5 Vissers LE, de Vries BB, Osoegawa $\mathrm{K}$ et al: Array-based comparative genomic hybridization for the genomewide detection of submicroscopic chromosomal abnormalities. Am J Hum Genet 2003; 73: 1261-1270.

6 Wagenstaller J, Spranger S, Lorenz-Depiereux B et al: Copy-number variations measured by single-nucleotide-polymorphism oligonucleotide arrays in patients with mental retardation. Am J Hum Genet 2007; 81: 768-779.

7 Girirajan S, Vlangos CN, Szomju BB et al: Genotype-phenotype correlation in SmithMagenis syndrome: evidence that multiple genes in $17 \mathrm{p} 11.2$ contribute to the clinical spectrum. Genet Med 2006; 8: 417-427.

8 Girirajan S, Williams SR, Garben JY, Nowak NJ, Hatchwell E, Elsea S: 17p11.2p12 triplication and del(17)q11.2q12 in a severely affected child with dup(17)p11.2p12 syndrome. Clin Genet 2007; 71: 1-12, In Press.

9 Ballif BC, Theisen A, Coppinger J et al: Expanding the clinical phenotype of the 3q29 microdeletion syndrome and characterization of the reciprocal microduplication. Mol Cytogenet 2008; $1: 8$.

10 Ballif BC, Theisen A, McDonald-McGinn DM et al: Identification of a previously unrecognized microdeletion syndrome of 16q11.2q12.2. Clin Genet 2008; 74: 469-475.

11 Shaffer LG, McCaskill C, Han JY et al: Molecular characterization of de novo secondary trisomy 13. Am J Hum Genet 1994; 55: 968-974.

12 Williams SR, Girirajan S, Tegay D, Nowak N, Hatchwell E, Elsea SH: Array comparative genomic hybridization of 52 subjects with a Smith-Magenis-like phenotype: identification of dosage-sensitive loci also associated with schizophrenia, autism, and developmental delay. J Med Genet 2009, PMID: 19752160.

13 Matos A, Nogueira A, Criado B, Pereira S, Castedo S, Montenegro N: Prenatal diagnosis of partial trisomy 2q. Case report. Prenat Diagn 1997; 17: 874-876.

14 Schumacher RE, Rocchini AP, Wilson GN: Partial trisomy 2q. Clin Genet 1983; 23 : 191-194.

15 Marchese SDG, Garver K, Stadler M: De novo 46,XX,dup(2)(q21-q31) ascertained by prenatal cytogenetic analysis. Am J Hum Genet 1984; 36: 103.

16 Farrell SA, Sajoo A, Maybury D, Speevak MD: Pure partial trisomy of 2q22-q23 secondary to a paternally inherited direct insertion: a rare duplication. Clin Genet 2003; 64: 255-257.

17 Nagase T, Kikuno R, Hattori A, Kondo Y, Okumura K, Ohara O: Prediction of the coding sequences of unidentified human genes. XIX. The complete sequences of 100 new cDNA clones from brain which code for large proteins in vitro. DNA Res 2000; 7: 347-355.

18 Roloff TC, Ropers HH, Nuber UA: Comparative study of methyl-CpG-binding domain proteins. BMC Genomics 2003; 4: 1

Supplementary Information accompanies the paper on European Journal of Human Genetics website (http://www.nature.com/ejhg) 